\title{
SOME GENERAL LAGRANGE INTERPOLATIONS OVER SIMPLEX FINITE ELEMENTS WITH REFERENCE TO DERIVATIVE SINGULARITIES
}

\author{
Masayuki OKABE \\ Mitsui Mining \& Smelting Co., Ltd., Tokyo, Japan \\ Noboru KIKUCHI* \\ Department of Mechanical Engineering and Applied Mechanics, The University of Michigan, USA
}

Received 6 February 1980

Revised manuscript received 22 September 1980

\begin{abstract}
For simplex finite elements, the native Lagrange family with arbitrarily placed nodes is presented in hierarchy-ranking expressions. It includes the well-known complete Lagrange family as well as the mid-edge Lagrange family to be proposed in this paper. This new family enables us to utilize harmonious combinations of interpolations of different orders in finite element analysis. As an application of developed simplex interpolations to fracture mechanics where some derivative singularities are needed, we then describe the semi-radial singularity mapping with examinations of peculiar trial function spaces.
\end{abstract}

\section{Introduction}

In Lagrange interpolation, a variety of finite element families has been proposed for 2- and 3-cubes, i.e. for squares and cuboids. For example, there is the regular Lagrange family due to Argyris et al. [1, 2], with nodes regularly spaced everywhere on the grid, and the mid-edge Lagrange family of Taylor [3] and others [4,5] composed of only vertex-type and side (or mid-edge) nodes. We note that the trial function spaces of their all members are fully known. Recently a general family for the cube called the native Lagrange family has been presented by the authors [5] with arbitrarily-placed nodes and including the regular and mid-edge families as subfamilies. The serendipity family by Zienkiewicz [4] is also well known, but only some lower serendipity elements are revealed as also being members of the native Lagrange family.

For simplex finite elements, on the other hand, only the complete Lagrange family with regularly placed nodes is fully recognized by Argyris [6, 7] and others $[4,8]$. However, it has been suggested that there may exist the mid-edge Lagrange family for a simplex, composed of only vertices and arbitrarily placed side nodes [9-11]. In addition, rather general finite elements have also been presented in the adaptive finite element procedures [12-14] utilizing not the conventional interpolation bases but the peculiar mixed bases [11] with both nodal and nodeless basis functions. It seems that potential exists to develop at least as many families for

*Formerly, TICOM, University of Texas at Austin, USA. 
simplexes as have already been found for cubes. In order to clarify such general simplex families, it is required that the interpolation elementology be reconstructed in a unified manner.

This paper first formulates the hierarchy-ranking bases associated with vertices and arbitrarily placed side nodes in volume coordinate expressions, which yield naturally the mid-edge Lagrange family for a simplex. Then the famous complete Lagrange family is recharacterized by its intrinsic hierarchy-ranking bases. Relaxing the constraints on the regularity of nodal placement, we shall further propose the native Lagrange family for simplex as a unifier of the complete and mid-edge families. This new, general family with arbitrarily-placed side and face nodes enable us to utilize compatible combinations of interpolations of different order. We also compare our native trial functions with other known interpolations such as the adaptive interpolations and collapsed rectangular polynomials.

The semi-radial singularity mapping is then described definitively so that the necessary derivative singularities are attained. The native simplex interpolations are thus easily and systematically applicable also to fracture mechanics, with only minor modification of the Jacobian transformation routine.

\section{Complete Lagrange family for simplex}

The well-known complete Lagrange family is composed of simplex finite elements with regularly placed nodes. For its triangular member of $M$ th order, Silvester [8] has presented an auxiliary expression for the shape function $N_{i}$ associated with node $i$ in the form

$$
N_{i}=\prod_{j=1}^{I}\left(M \omega_{1}-j+1\right) / j \prod_{j=1}^{J}\left(M \omega_{2}-j+1\right) / j \prod_{j=1}^{K}\left(M \omega_{3}-j+1\right) / j,
$$

where

$$
I=M \omega_{1}(i), \quad J=M \omega_{2}(i), \quad K=M \omega_{3}(i) .
$$

Here $\omega_{k}$ denotes the 2-D volume (i.e. area) coordinate associated with vertex $k$, and $\omega_{k}(i)$ is its value at node $i$. Notice that

$$
\prod_{j=1}^{0}\left(M \omega_{k}-j+1\right) / j=1 .
$$

According to Argyris et al. [7], it is easy to rewrite eq. (1) in the Lagrangian interpolation polynomial form as

$$
N_{i}=\prod_{k=1}^{3} Q_{i}\left(\omega_{k}\right)
$$

where

$$
Q_{i}\left(\omega_{k}\right)=\prod_{\substack{j \\ \omega_{k}(j)<\omega_{k}(i)}}\left\{\omega_{k}-\omega_{k}(j)\right\} /\left\{\omega_{k}(i)-\omega_{k}(j)\right\}
$$




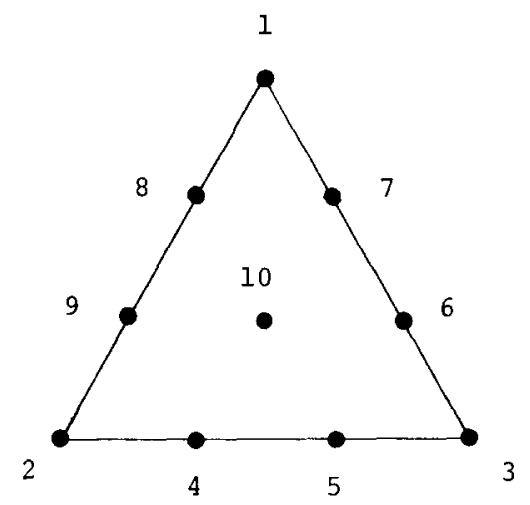

Fig. 1. Cubic element geometry of 10 -node triangle.

It is noted that this element contains the complete polynomial set of degree $M$, and hence the complete Lagrange family for simplex corresponds naturally to the serendipity family for a cube. From the standpoint of the nodal placement regularity, it further corresponds to the regular Lagrange family for cube.

Consider, for instance, the cubic triangle in fig. 1. Then immediately eq. (4) yields the shape functions in table 1 . Zero lines of some typical shape functions are illustrated in fig. 2 . We note that eq. (1) implies regular nodal placement, while eq. (4) is, in essence, free from such regularity. Apparently, eq. (4) is applicable to any arbitrary nodal placement.

Let us apply eq. (4) to the 9-node triangular element in fig. 3, extinguishing the centroidal node of preceding cubic triangle. Then all the cubic shape functions related to vertices and side nodes are again produced. It is, however, noted that such a trial function does not exhibit polynomial completeness, and hence it cannot be accepted in the finite element analysis. The application of eq. (4) should, therefore, be restricted to the complete Lagrange elements.

Table 1

Shape functions $N_{i}$ for 10 - and 9-node triangular elements in figs. 1 and 3 , respectively.

\begin{tabular}{cll}
\hline $\begin{array}{c}\text { Node } \\
i\end{array}$ & \multicolumn{1}{c}{$\begin{array}{c}\text { Ten-node triangle } \\
N_{i}^{(10)}\end{array}$} & \multicolumn{1}{c}{$\begin{array}{c}\text { Nine-node triangle } \\
N_{i}^{(9)}\end{array}$} \\
\hline 1 & $\omega_{1}\left(3 \omega_{1}-1\right)\left(3 \omega_{1}-2\right) / 2$ & $\omega_{1}\left\{\left(3 \omega_{1}-1\right)\left(3 \omega_{1}-2\right)-9 \omega_{2} \omega_{3}\right\} / 2$ \\
2 & $\omega_{2}\left(3 \omega_{2}-1\right)\left(3 \omega_{2}-2\right) / 2$ & $\omega_{2}\left\{\left(3 \omega_{2}-1\right)\left(3 \omega_{2}-2\right)-9 \omega_{3} \omega_{1}\right\} / 2$ \\
3 & $\omega_{3}\left(3 \omega_{3}-1\right)\left(3 \omega_{3}-2\right) / 2$ & $\omega_{3}\left\{\left(3 \omega_{3}-1\right)\left(3 \omega_{3}-2\right)-9 \omega_{1} \omega_{2}\right\} / 2$ \\
4 & $9 \omega_{2} \omega_{3}\left(3 \omega_{2}-1\right) / 2$ & $9 \omega_{2} \omega_{3}\left(3 \omega_{2}-3 \omega_{3}+1\right) / 4$ \\
5 & $9 \omega_{2} \omega_{3}\left(3 \omega_{3}-1\right) / 2$ & $9 \omega_{2} \omega_{3}\left(3 \omega_{3}-3 \omega_{2}+1\right) / 4$ \\
6 & $9 \omega_{3} \omega_{1}\left(3 \omega_{3}-1\right) / 2$ & $9 \omega_{3} \omega_{1}\left(3 \omega_{3}-3 \omega_{1}+1\right) / 4$ \\
7 & $9 \omega_{3} \omega_{1}\left(3 \omega_{1}-1\right) / 2$ & $9 \omega_{3} \omega_{1}\left(3 \omega_{1}-3 \omega_{3}+1\right) / 4$ \\
8 & $9 \omega_{1} \omega_{2}\left(3 \omega_{1}-1\right) / 2$ & $9 \omega_{1} \omega_{2}\left(3 \omega_{1}-3 \omega_{2}+1\right) / 4$ \\
9 & $9 \omega_{1} \omega_{2}\left(3 \omega_{2}-1\right) / 2$ & $9 \omega_{1} \omega_{2}\left(3 \omega_{2}-3 \omega_{1}+1\right) / 4$ \\
10 & $27 \omega_{1} \omega_{2} \omega_{3}$ & \\
\hline
\end{tabular}



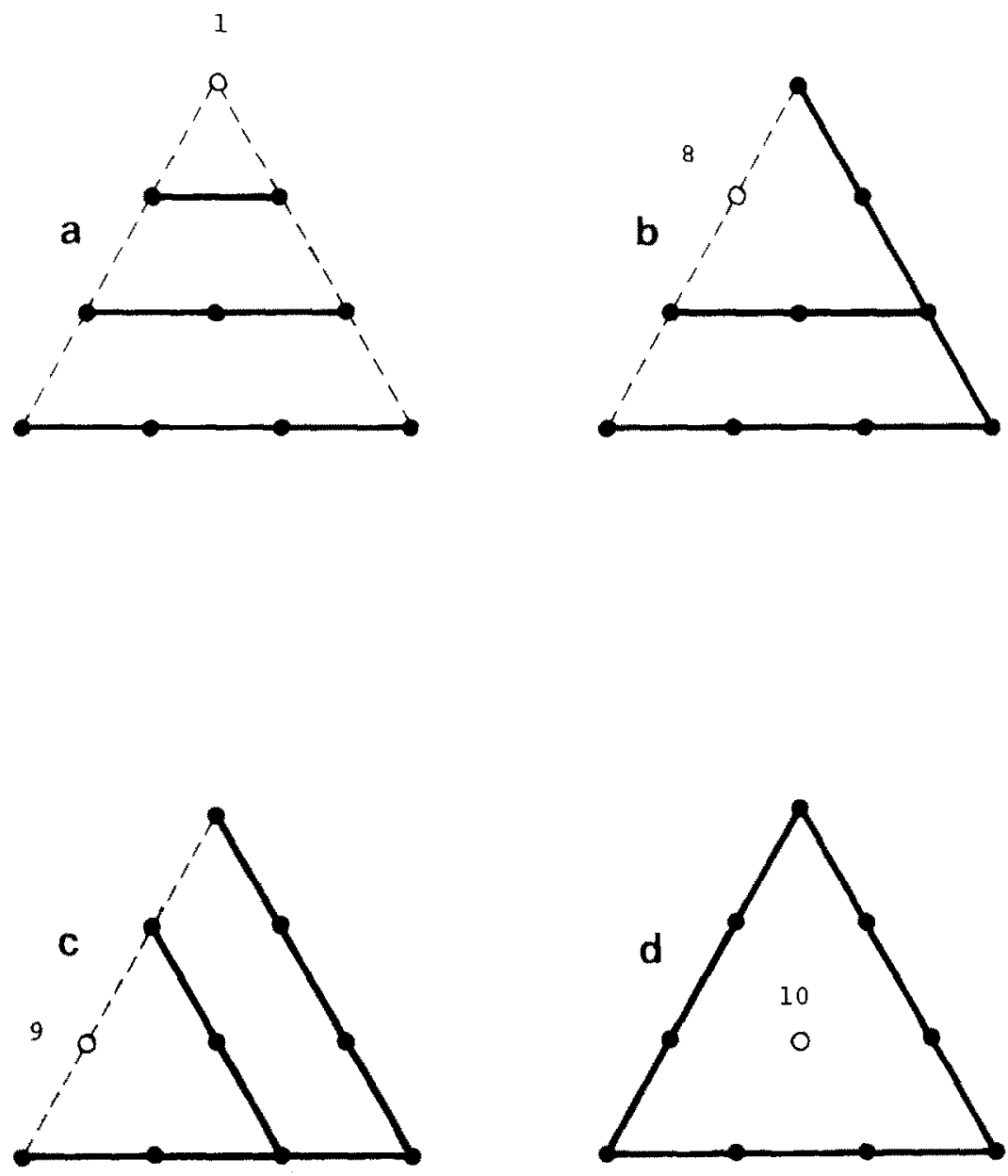

Fig. 2. Zero lines of typical shape functions in cubic triangle; (a) $N_{1}=0$, (b) $N_{8}=0$, (c) $N_{9}=0$, (d) $N_{10}=0$.

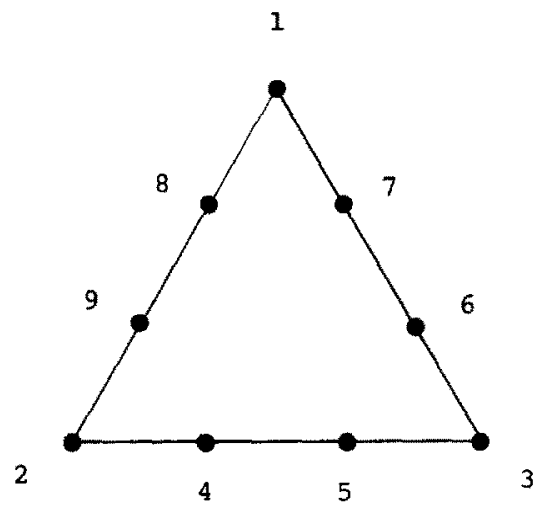

Fig. 3. 9-node triangular element without a centroidal node. 


\section{Trial function space and in-family relevancy}

We shall describe here how suitable shape functions are derived for the 9-node triangle so that quadratics can be exactly interpolated.

Consider two finite elements of $n$ and $n-1$ nodes with the same geometry, where node $j$ of the $n$-node element disappears in the other. Then corresponding trial functions can be expressed

$$
\phi^{(n)}=\sum_{i=1}^{n} \phi_{i}^{(n)} N_{i}^{(n)}
$$

and

$$
\phi^{(n-1)}=\sum_{i \neq j} \phi_{i}^{(n-1)} N_{i}^{(n-1)} .
$$

Here $\phi_{i}$ is the $\phi$-value at node $i$.

Let $f$ and $f_{i}$ denote an arbitrary function and its nodal value at node $i$, respectively. Then the trial function space $\mathscr{F}^{(n)}$ for $n$-node element can be defined as

$$
\mathscr{F}^{(n)}=\left\{f: E_{\mathscr{F}}^{(n)}(f)=f-\sum_{i=1}^{n} f_{i} N_{i}^{(n)}=0\right\} .
$$

Similarly the other trial function space $\mathscr{F}^{(n-1)}$ can be written

$$
\mathscr{F}^{(n-1)}=\left\{f: E_{\mathscr{F}}^{(n-1)}(f)=f-\sum_{i \neq j} f_{i} N_{i}^{(n-1)}=0\right\} .
$$

Suppose that the $\mathscr{F}^{(n)}$ space includes (9) as a subspace. Then the $\phi^{(n)}$ trial function should be capable of identity with $\phi^{(n-1)}$ trial function, but this is only realized under pair of the reducible conditions of the form

$$
\phi_{i}^{(n)}=\phi_{i}^{(n-1)}, \quad(i \neq j)
$$

and

$$
\phi_{j}^{(n)}=\sum_{i \neq j} \phi_{i}^{(n-1)} N_{i}^{(n-1)}(j)
$$

From eqs. (6), (7), (10) and (11), we have

$$
N_{i}^{(n)}=N_{i}^{(n-1)}-N_{i}^{(n-1)}(j) N_{j}^{(n)} \quad(i \neq j) .
$$

Thus naturally we can regard these two elements as members of the same family, provided that eq. (12) is realized. Hence, eq. (12) is termed the in-family relevancy [5]. 
The simplest family member has, in its most general form, the so-called linear interpolation basis, enabling the unknown $\phi$ to vary linearly on all the boundary edges. So, the $\mathscr{F}^{(n)}$ space is required to include a linear trial function space also. We refer to this as linear inclusiveness. The reader can easily develop the appropriate in-family relevancy expression by applying eq. (12) recurrently.

Consider then previous 10- and 9-node triangular elements in figs. 1 and 3, respectively. Following up the suggestion by Ciarlet and Raviart [9], Mitchell and Wait [10] have shown now the tenth parameter due to the centroid of triangle can be eliminated, using the linear relation by

$$
\phi_{10}^{(10)}=a \sum_{i=1}^{3} \phi_{i}^{(9)}+b \sum_{i=4}^{9} \phi_{i}^{(9)}
$$

where $a$ and $b$ denote appropriate coefficients to be determined. Then immediately we have

$$
\begin{array}{ll}
N_{i}^{(9)}=N_{i}^{(10)}+a N_{10}^{(10)} & (1 \leq i \leq 3) \\
N_{i}^{(9)}=N_{i}^{(10)}+b N_{10}^{(10)} & (4 \leq i \leq 9) .
\end{array}
$$

The in-family relevancy by eq. (12) further prescribes that the coefficients should satisfy

$$
a=N_{i}^{(9)}(10), \quad(1 \leq i \leq 3), \quad b=N_{i}^{(9)}(10) \quad(4 \leq i \leq 9) .
$$

Thus evidently eq. (13) is identified with the reducible condition (11).

However, eq. (14) is insufficient to determine the $N_{i}^{(9)}$ shape functions uniquely, since the $N_{i}^{(9)}$-value at node 10 is included. Thus, finite element families cannot be defined by the in-family relevancy alone.

We suppose here that the $\phi^{(9)}$ trial function interpolates quadratics exactly. Then we have

$$
E_{F}^{(9)}(1)=(1-3 a-6 b) N_{10}^{(10)}=0, \quad E_{\mathscr{F}}^{(9)}\left(\omega_{i}\right)=(1-3 a-6 b) N_{10}^{(10)} / 3=0,
$$

and

$$
E_{\Im}^{(9)}\left(\omega_{i} \omega_{j}\right)=\left\{1-4 b-3(3 a+2 b) \delta_{i j}\right\} N_{10}^{(10)} / 9=0
$$
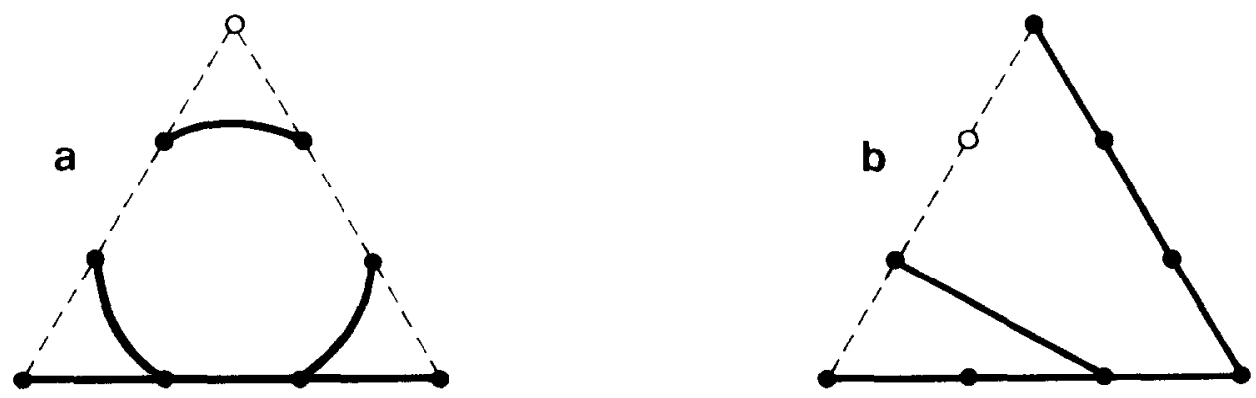

Fig. 4. Zero lines of typical shape functions in 9-node triangle; (a) $N_{1}=0$, (b) $N_{8}=0$. 
Here $\delta_{i j}$ denotes the Kronecker delta. The coefficients are thus uniquely determined as

$$
a=-1 / 6, \quad b=1 / 4 .
$$

The shape functions for 9-node triangle obtained are given in table 1, and typical zero lines are illustrated in fig. 4.

This example strongly suggests the presence of some general simplex family composed of only vertices and side nodes, which is analogous to the mid-edge Lagrange family for a cube. It should be, however, noted that the corresponding cubic error is

$$
E_{F}^{(9)}\left(\omega_{i} \omega_{j} \omega_{k}\right)=\left\{\begin{array}{rr}
\omega_{1} \omega_{2} \omega_{3} & (i=j=k) \\
-\omega_{1} \omega_{2} \omega_{3} & (i=j \neq k) \\
\omega_{1} \omega_{2} \omega_{3} & (i \neq j \neq k)
\end{array}\right.
$$

and consequently no cubic monomials are reproducible. Thus we cannot define the mid-edge Lagrange family for a simplex by prescribing monomial bases.

\section{Hierarchy-ranking basis}

In familizing the rectangular finite elements, we have demonstrated elsewhere [5] the significance of the hierarchy-ranking bases. Before simplex familization we shall describe the hierarchy-ranking concept briefly.

The relevant trial function space $\mathscr{F}$ can be uniquely characterized by the so-called interpolation basis $N=\left\{N_{i}\right\}$, which is composed of the shape functions $N_{i}$ satisfying

$$
N_{i}(j)=\delta_{i j}
$$

Naturally the same trial function space is definable by another arbitrary basis $\boldsymbol{S}=\left\{\boldsymbol{S}_{i}\right\}$. Evidently the $S_{i}$ function is a component of the $\mathscr{F}$ space, and hence we have

$$
E_{\mathscr{F}}\left(S_{i}\right)=S_{i}-\sum_{j} S_{i}(j) N_{j}=0,
$$

or in matrix form

$$
\left\{S_{i}\right\}=\left[S_{i}(j)\right]\left\{N_{i}\right\} .
$$

It is thus obvious that if we prescribe the trial function space $\mathscr{F}$ by the arbitrary basis $S$, the interpolation basis $\boldsymbol{N}$ can then easily be obtained through the basis transformation (21), provided that the constant transformation matrix in eq. (21) is non-singular.

We decompose the $\boldsymbol{N}$ and $\boldsymbol{S}$ bases according to nodal rank, which is defined as 0,1 and 2 for vertex-type, side and face nodes, respectively. Then eq. (21) can be rewritten

$$
\left\{\begin{array}{l}
S_{0} \\
S_{1} \\
S_{2}
\end{array}\right\}=\left[\begin{array}{lll}
S_{0}(0) & S_{0}(1) & S_{0}(2) \\
S_{1}(0) & S_{1}(1) & S_{1}(2) \\
S_{2}(0) & S_{2}(1) & S_{2}(2)
\end{array}\right]\left\{\begin{array}{l}
N_{0} \\
N_{1} \\
N_{2}
\end{array}\right\}
$$


where suffix indicates the nodal rank. We impose further the ranking conditions of the form

$$
\begin{aligned}
& \boldsymbol{S}_{i}(j)=\mathbf{0} \quad(i>j) \\
& \boldsymbol{S}_{i}(i)=\boldsymbol{I}_{i} .
\end{aligned}
$$

Here $\boldsymbol{I}_{i}$ stands for the appropriate unit matrix. The ranking basis satisfying eqs. (23) and (24) yields immediately

$$
\left\{\begin{array}{l}
\boldsymbol{S}_{0} \\
\boldsymbol{S}_{1} \\
\boldsymbol{S}_{2}
\end{array}\right\}=\left[\begin{array}{ccc}
\boldsymbol{I}_{0} & \boldsymbol{S}_{0}(1) & \boldsymbol{S}_{0}(2) \\
0 & \boldsymbol{I}_{1} & \boldsymbol{S}_{1}(2) \\
0 & 0 & \boldsymbol{I}_{2}
\end{array}\right]\left[\begin{array}{l}
\boldsymbol{N}_{0} \\
\boldsymbol{N}_{1} \\
\boldsymbol{N}_{2}
\end{array}\right\} .
$$

The interpolation basis can then be methodically obtained in descending order of rank without matrix inversion as

$$
\boldsymbol{N}_{2}=\boldsymbol{S}_{2}, \quad \boldsymbol{N}_{1}=\boldsymbol{S}_{1}-\boldsymbol{S}_{1}(2) \boldsymbol{N}_{2}, \quad \boldsymbol{N}_{0}=\boldsymbol{S}_{0}-\boldsymbol{S}_{0}(1) \boldsymbol{N}_{1}-\boldsymbol{S}_{0}(2) \boldsymbol{N}_{2}
$$

If we have no face nodes, then eq. (26) can simply be rewritten

$$
\boldsymbol{N}_{1}=\boldsymbol{S}_{1}, \quad \boldsymbol{N}_{0}=\boldsymbol{S}_{0}-\boldsymbol{S}_{0}(1) \boldsymbol{N}_{1}
$$

Note that the well-known linear interpolation basis should be adopted as the vertex ranking subbasis $\boldsymbol{S}_{0}$. Then the linear trial function is trivially contained in our trial function space.

We further impose the spatial hierarchy [5]. Namely, after substitution of a surface equation of our 2-simplex element, the basis functions related to nodes on the surface should be equivalent to the famous Lagrangian interpolation polynomials, while the others should be zero. Such ranking basis as realizes the spatial hierarchy is, in general, termed the hierarchyranking basis. Any Lagrange families for simplex can then be expected to be uniquely characterized by their intrinsic hierarchy-ranking bases, as realized in Lagrange families for the cube.

\section{1-D Lagrange family in volume coordinate expressions}

It has been shown how that the conventional Lagrangian interpolation polynomials constitute the 1-D Lagrange family $[5,11]$. We shall recharacterize that simple family using volume coordinate expressions.

Consider first the $n$-node bar element in fig. 5 along the usual $\xi$-axis. The ranking basis functions of nodal rank 1 are naturally identical to the appropriate Lagrangian interpolation polynomials so that

$$
S_{i}=\left[\prod_{j \neq i} L_{i}^{j}\right]\left(1-\xi^{2}\right) /\left(1-\xi_{i}^{2}\right) \quad(i=3, \ldots, n)
$$




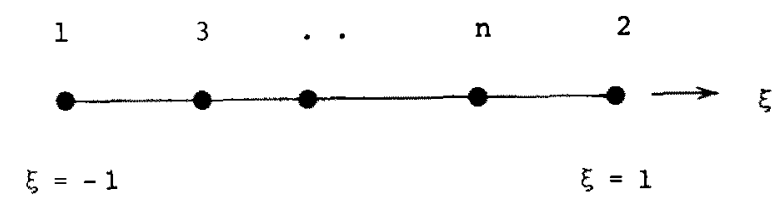

Fig. 5. 1-D Lagrange element of $n$-node bar.

Here $L_{i}^{j}$ denotes the Lagrangian interpolation unit of rank 1 at node $i$ and $j$ and is given by

$$
L_{i}^{j}=\left(\xi-\xi_{j}\right) /\left(\xi_{i}-\xi_{j}\right)
$$

The ranking basis functions for vertices, on the other hand, can be written

$$
S_{1}=(1-\xi) / 2, \quad S_{2}=(1+\xi) / 2 .
$$

Let us introduce the 1-D volume coordinates $\omega_{1}$ and $\omega_{2}$ associated with vertices 1 and 2 , respectively. Then naturally eq. (30) can be rewritten

$$
S_{i}=\omega_{i}, \quad(i=1,2) .
$$

Thus in order to recharacterize the 1-D Lagrange family, the Lagrangian interpolation unit by eq. (29) must be formulated in volume coordinate expressions.

Evidently there exist several candidates for the Lagrangian interpolation unit in volume coordinates. For example, we have

$$
\begin{aligned}
& L_{i}^{j}=\left\{\omega_{1}-\omega_{1}(j)\right\} /\left\{\omega_{1}(i)-\omega_{1}(j)\right\} \\
& L_{i}^{j}=\left\{\omega_{2}-\omega_{2}(j)\right\} /\left\{\omega_{2}(i)-\omega_{2}(j)\right\} \\
& L_{i}^{j}=\left\{\omega_{2}(j) \omega_{1}-\omega_{1}(j) \omega_{2}\right\} /\left\{\omega_{2}(j) \omega_{1}(i)-\omega_{1}(j) \omega_{2}(i)\right\} \\
& L_{i}^{i}=\left\{\omega_{1}-\omega_{2}-\omega_{1}(j)+\omega_{2}(j)\right\} /\left\{\omega_{1}(i)-\omega_{2}(i)-\omega_{1}(j)+\omega_{2}(j)\right\}
\end{aligned}
$$

Although these Lagrangian interpolation units are all equivalent in one dimension, eqs. (32) to (35) yield quite different basis functions in higher dimensions. Therefore, we must select the best Lagrangian interpolation unit so that the definitive law for nodal rank 1 is adequate in any dimension. In conclusion, the Lagrangian interpolation unit in eq. (35) is most preferable as shall be shown in the following section.

\section{Mid-edge Lagrange family for simplex}

We shall consider here 2-simplex finite elements composed of only vertices and side nodes, in order to select the best Lagrangian interpolation unit. 
Let nodes $i$ and $j$ be of nodal rank 1 on an edge due to vertices $p$ and $q$. Then noting eq. (28), the ranking basis function $S_{i}$ can be written

$$
S_{i}=\left[\prod_{i \neq i} L_{i}^{j}\right] \omega_{p} \omega_{q} / \omega_{p}(i) \omega_{q}(i)
$$

where $\omega_{p}$ and $\omega_{q}$ denote 2-D volume coordinates associated with vertices $p$ and $q$, respectively. Let us apply eq. (36) to $S_{8}$ for the preceding 9-node triangle in fig. 3. Then the Lagrangian interpolation units in eqs. (32) to (35) yield quite different basis functions, and we find that only the Lagrangian interpolation unit of eq. (35) yields $N_{8}^{(9)}$ in table 1 . Zero lines of $S_{8}$ due to eqs. (32) to (34) are illustrated in fig. 6 , for comparison.

The hierarchy-ranking subbasis of nodal rank 1 can thus be defined by eq. (36) with the Lagrangian interpolation unit of the form

$$
L_{i}^{j}=\left\{\omega_{p}-\omega_{q}-\omega_{p}(j)+\omega_{q}(j)\right\} /\left\{\omega_{p}(i)-\omega_{q}(i)-\omega_{p}(j)+\omega_{q}(j)\right\} .
$$

Naturally the linear field must be reproducible in the relevant trial functions, and therefore we have for vertices' basis functions

$$
S_{i}=\omega_{i} \quad(i=1,2,3)
$$
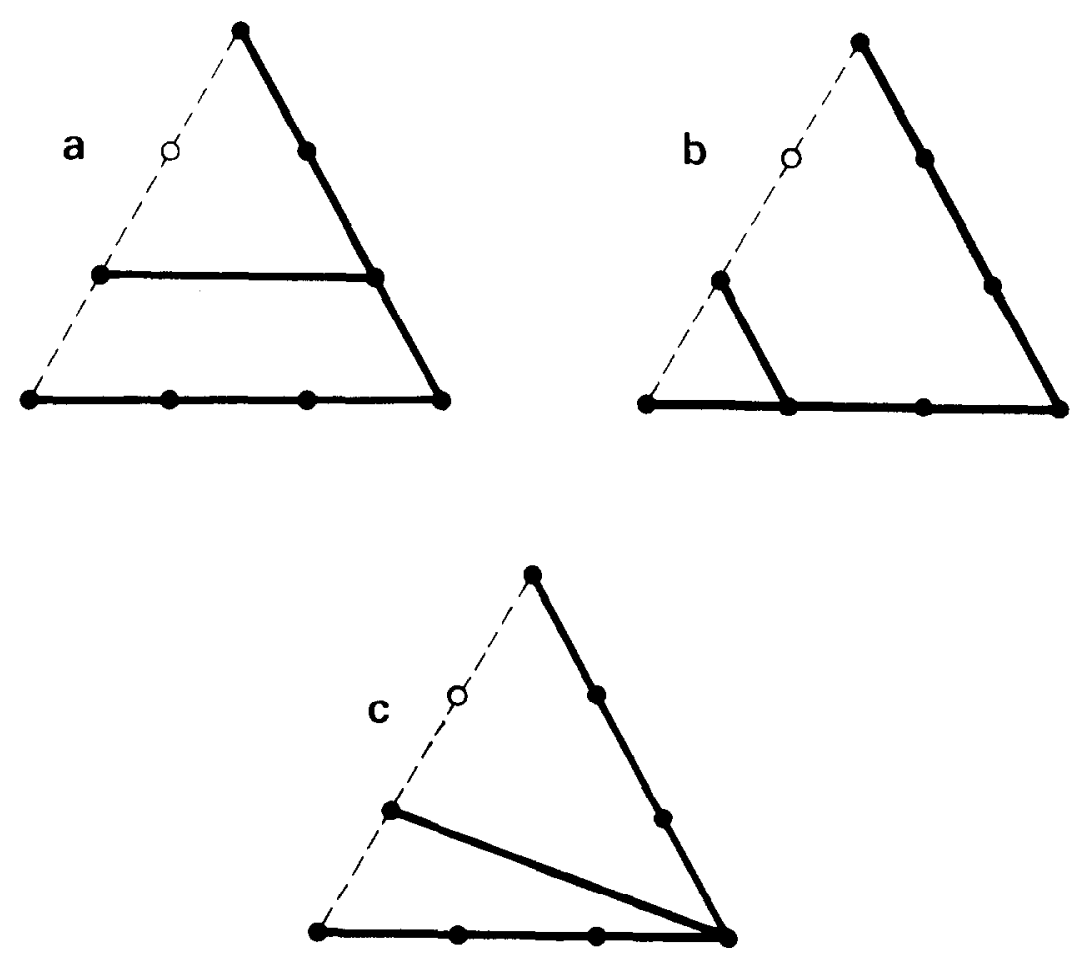

Fig. 6. Zero lines of the ranking basis function $S_{8}$ in the 9-node triangle, due to different Lagrangian interpolation units; (a) $S_{8}=0$ due to $L_{i}^{j}$ by eq. (32), (b) $S_{8}=0$ due to $L_{i}^{j}$ by eq. (33), (c) $S_{8}=0$ due to $L_{i}^{j}$ by eq. (34). 


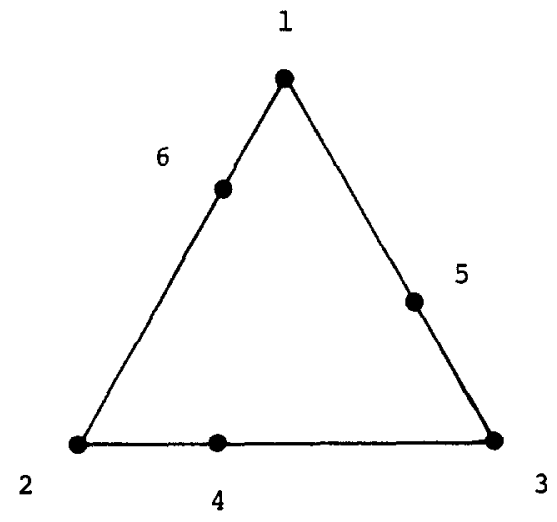

Fig. 7. Quadratic mid-edge triangle with arbitrarily placed side nodes.

The reader can then easily verify that eqs. (36) to (38) readily yield the interpolation basis in table 1 for the above 9-node element.

We emphasize here that eq. (36) with eq. (37) is essentially free from the regularity of side-node placement. The mid-edge Lagrange family for simplex composed of only vertices and arbitrarily placed side nodes can thus be defined by eqs. (36) to (38) in hierarchy-ranking expressions.

Consider, for example, a quadratic mid-edge element in fig. 7 . Then we have immediately

$$
S_{4}=\omega_{2} \omega_{3} / \omega_{2}(4) \omega_{3}(4), \quad S_{5}=\omega_{3} \omega_{1} / \omega_{3}(5) \omega_{1}(5), \quad S_{6}=\omega_{1} \omega_{2} / \omega_{1}(6) \omega_{2}(6) .
$$

For vertices, eq. (38) is adequate. It is then easy to verify that this trial function interpolates quadratics exactly [11].

Introduce here the existence parameter $\kappa_{i}$ related to side node $i$ by

$$
\kappa_{i}= \begin{cases}1, & \text { if node } i \text { exists, } \\ 0, & \text { if node } i \text { disappears }\end{cases}
$$

Then we can easily develop the variable-node trial function $[5,15]$ for our quadratic member such that

$$
\begin{aligned}
& N_{1}=\omega_{1}-\omega_{1}(5) N_{5}-\omega_{1}(6) N_{6}, \quad N_{2}=\omega_{2}-\omega_{2}(6) N_{6}-\omega_{2}(4) N_{4}, \\
& N_{3}=\omega_{3}-\omega_{3}(4) N_{4}-\omega_{3}(5) N_{5}, \quad N_{4}=\kappa_{4} \omega_{2} \omega_{3} / \omega_{2}(4) \omega_{3}(4), \\
& N_{5}=\kappa_{5} \omega_{3} \omega_{1} / \omega_{3}(5) \omega_{1}(5), \quad N_{6}=\kappa_{6} \omega_{1} \omega_{2} / \omega_{1}(6) \omega_{2}(6)
\end{aligned}
$$

We remark that the definitive laws in eqs. (36) to (38) take the same form in any dimension. For example, in one dimension, the famous 1-D Lagrange family is reproduced but in volume coordinate expressions. Then the spatial hierarchy inherent to the volume coordinates [5] guarantees the satisfaction of the spatial hierarchy for all the mid-edge simplex members. Thus if we substitute an edge equation for all the hierarchy-ranking basis functions, then the basis functions related to nodes on the edge are equivalent to the Lagrangian interpolation 
polynomials, while the others not in the surface are zero. The mid-edge Lagrange family for simplex is thus $C_{0}$ conformable.

However, as predicted by Ciarlet and Raviart [9], our mid-edge trial function space observe polynomial completeness of degree two at most. In finite element applications, we must keep this feature in mind.

It is further emphasized that we fully relax the constraint on the regularity of side-node placement in our mid-edge simplex family. Although such relaxation is meaningless in finite element analysis [11], it is essentially needed in other surface generation fields.

\section{Recharacterization of the complete Lagrange family for simplex}

We treat again here the famous complete Lagrange family for simplex in hierarchy-ranking expressions.

It is first noted in rectangular familizations that the hierarchy-ranking subbases of nodal rank 0 and 1 are common to the regular and mid-edge Lagrange families for cube [5]. Naturally this feature should be preserved in simplex familizations also, and hence eqs. (36) to (38) are always applicable to the hierarchy-ranking basis functions associated with vertices and side nodes.

We then note that the hierarchy-ranking subbasis of maximum nodal rank is to be identical to the corresponding interpolation subbasis as shown in eq. (26). We can thus utilize eqs. (4) and (5) such that

$$
S_{i}=\prod_{k=1}^{3} Q_{i}\left(\omega_{k}\right)
$$

Here node $i$ is of nodal rank 2, and $Q_{i}$ is given by eq. (5).

Consider, for instance, a cubic complete triangle in fig. 1 . Then eq. (42) yields immediately

$$
S_{10}=27 \omega_{1} \omega_{2} \omega_{3} .
$$

Applying eq. (36) for side nodes, we have further

$$
\begin{array}{ll}
S_{4}=9 \omega_{2} \omega_{3}\left(3 \omega_{2}-3 \omega_{3}+1\right) / 4, & S_{5}=9 \omega_{2} \omega_{3}\left(3 \omega_{3}-3 \omega_{2}+1\right) / 4, \\
S_{6}=9 \omega_{3} \omega_{1}\left(3 \omega_{3}-3 \omega_{1}+1\right) / 4, & S_{7}=9 \omega_{3} \omega_{1}\left(3 \omega_{1}-3 \omega_{3}+1\right) / 4, \\
S_{8}=9 \omega_{1} \omega_{2}\left(3 \omega_{1}-3 \omega_{2}+1\right) / 4, & S_{9}=9 \omega_{1} \omega_{2}\left(3 \omega_{2}-3 \omega_{1}+1\right) / 4
\end{array}
$$

For vertices, eq. (38) is valid also in this example. The reader can then easily verify that this element is identical to the well-known cubic triangle.

We have thus succeeded in recharacterising the complete Lagrange family for a simplex by its intrinsic hierarchy-ranking bases. Evidently in eq. (42), the ranking basis functions of nodal rank 2 give identically zero functions on the boundaries, and hence the spatial hierarchy of the complete Lagrange members is guaranteed. 


\section{Native Lagrange family for simplex with arbitrarily placed nodes}

Attention is drawn to the fact that the definitive laws of the hierarchy-ranking subbases due to vertices and side nodes are common to the complete and mid-edge simplex families, which suggests strongly the existence of a more general simplex family with arbitrarily placed nodes. In such a general family, the hierarchy-ranking basis functions for vertices and side nodes are naturally defined by eqs. (36) to (38) as occurs in the rectangular case. Therefore, our objective here is to find the definitive law for arbitrarily placed face nodes.

Let node $i$ be of nodal rank 2 . Then using nodes $j$ and $l(l<j)$ of rank 2 , the $Q_{i}$ function of eq. (5) can be redesignated

$$
P_{i}\left(\omega_{k}\right)=\left[\prod_{\substack{j \\ \omega_{k}(j)<\omega_{k}(i) \\ \omega_{k}(j) \neq \omega_{k}(l) \forall l<j}}\left\{\omega_{k}-\omega_{k}(j)\right\} /\left\{\omega_{k}(i)-\omega_{k}(j)\right\}\right] \omega_{k} / \omega_{k}(i) .
$$

Then eq. (42) can be restated as

$$
S_{i}=\prod_{k=1}^{3} P_{i}\left(\omega_{k}\right)
$$

Notice that eq. (46) with eq. (45) is now free from the face-node regularity, avoiding any multiple roots.

Thus utilizing eqs. (36) to (38) for vertices and side nodes, we have a new, general finite element family in hierarchy-ranking expressions, which allows arbitrarily placed side and face nodes. This family is termed here the native Lagrange family for simplex, since it is based on the unsophisticated, native transformation of eq. (45).

Consider, for example, the 5-node native element shown in fig. 8 . Then for node 5 of rank 2 , eqs. (45) and (46) yield

$$
S_{5}=27 \omega_{1} \omega_{2} \omega_{3}
$$

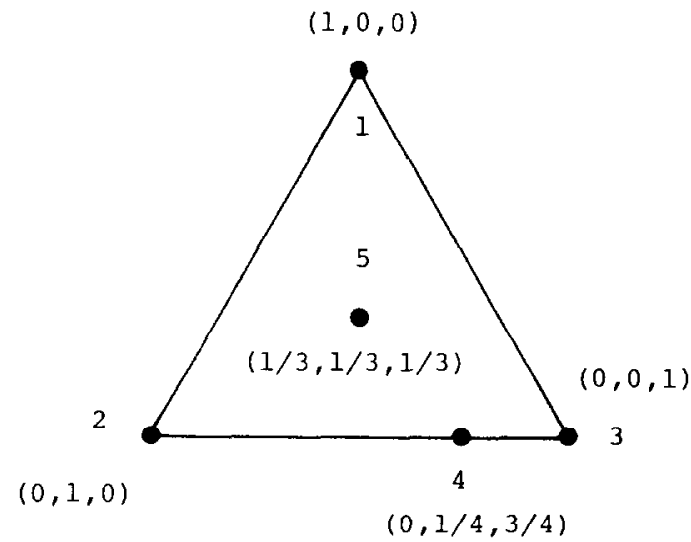

Fig. 8. 5-node native element in the volume coordinates $\left(\omega_{1}, \omega_{2}, \omega_{3}\right)$. 
For node 4 of rank 1, we have

$$
S_{4}=16 \omega_{2} \omega_{3} / 3
$$

Thus with eq. (38), the basis transformation (25) yields immediately the upper triangular scheme

$$
\left\{\begin{array}{l}
S_{1} \\
S_{2} \\
S_{3} \\
S_{4} \\
S_{5}
\end{array}\right\}=\left[\begin{array}{ccccc}
1 & 0 & 0 & 0 & 1 / 3 \\
0 & 1 & 0 & 1 / 4 & 1 / 3 \\
0 & 0 & 1 & 3 / 4 & 1 / 3 \\
0 & 0 & 0 & 1 & 16 / 27 \\
0 & 0 & 0 & 0 & 1
\end{array}\right]\left\{\begin{array}{c}
N_{1} \\
N_{2} \\
N_{3} \\
N_{4} \\
N_{5}
\end{array}\right\}
$$

Solving eq. (49), the interpolation basis is given by

$$
\begin{aligned}
& N_{1}=\omega_{1}\left(1-9 \omega_{2} \omega_{3}\right), \quad N_{2}=\omega_{2}\left(3-4 \omega_{3}-15 \omega_{3} \omega_{1}\right) / 3, \\
& N_{3}=\omega_{3}\left(1-4 \omega_{2}+3 \omega_{1} \omega_{2}\right), \quad N_{4}=16 \omega_{2} \omega_{3}\left(1-3 \omega_{1}\right) / 3, \quad N_{5}=27 \omega_{1} \omega_{2} \omega_{3} .
\end{aligned}
$$

On the other hand, if we use the $Q_{i}$ function of eq. (5) in place of the $P_{i}$ function in eq. (46), we have instead

$$
S_{5}=81 \omega_{1} \omega_{2} \omega_{3}\left(4 \omega_{2}-1\right)
$$

It is thus obvious that the hierarchy-ranking subbases of rank 2 should be defined by using only the face nodes. Otherwise, we have rather higher order polynomials than are warranted, needlessly complicating the interpolation.

We note that the $P_{i}$ function in eq. (45) is identical to the $Q_{i}$ function of eq. (5) in case of the regularly placed nodes. The native Lagrange family for a simplex thus naturally includes the complete and mid-edge families as subfamilies. Furthermore, since the definition, (46), of the hierarchy-ranking basis function gives identically zero on the boundaries, all the native simplex members are $\mathrm{C}_{0}$ conformable.

In the practical finite element applications, it has been expected that the use of rather higher order approximations with compatible combinations of lower order elements may improve the efficiency a great deal. In fact, this harmony occurs in the adaptive finite element procedures [12-14] as schematically shown in fig. 9, but utilizing the mixed bases with the so-called nodeless variables. It should be, however, noted that such mixed bases are proved to be theoretically equivalent to the conventional interpolation bases in finite element analysis [11]. We shall demonstrate here the identities of adaptive finite elements and native simplex members.

Consider a typical transitive element shown in fig. 10. Then the adaptive basis function associated with the side degree of freedom is nodelessly expressed as

$$
S_{4}=\omega_{2} \omega_{3} .
$$

For vertices, eq. (38) is adopted also in the adaptive elementology. 


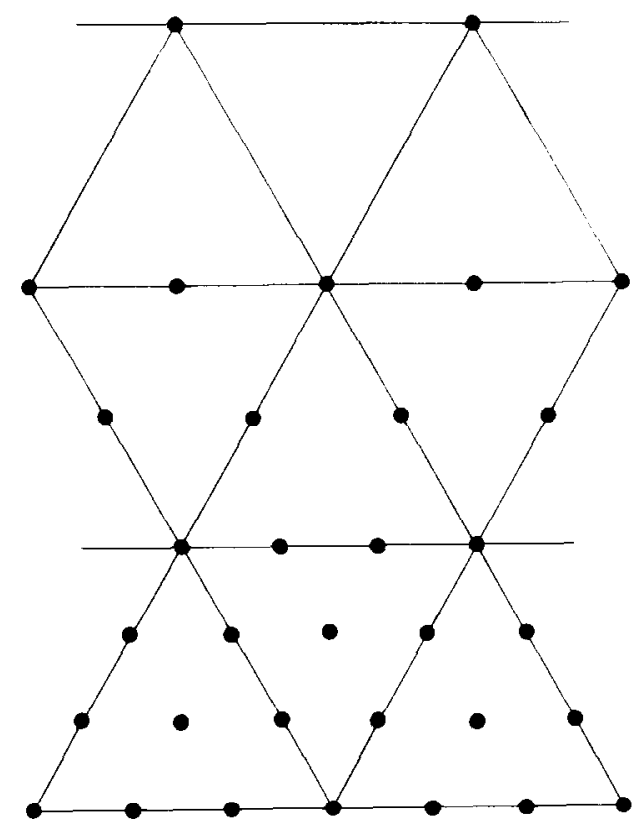

Fig. 9. Compatible combination of different order finite elements. In adaptive finite element procedure, nodeless basis functions are attached to side and internal degrees of freedom without placement of side and face nodes.

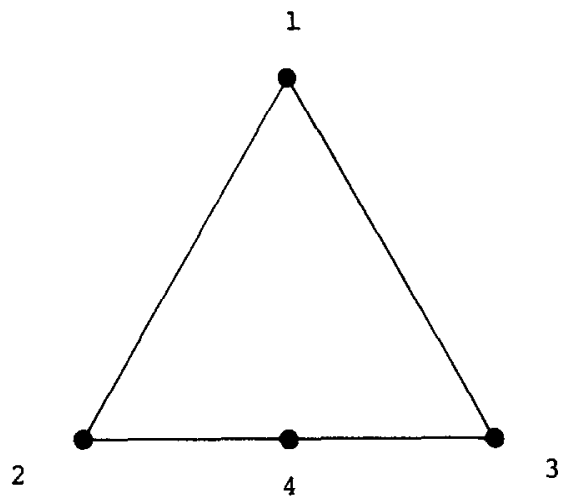

Fig. 10. Typical transitive finite element combining the linear and quadratic complete triangles conformably.

Let us assume node 4 at the centre of the concerning edge $\overline{23}$. Then the basis transformation (21) can again be used, and we have

$$
\left\{\begin{array}{l}
S_{1} \\
S_{2} \\
S_{3} \\
S_{4}
\end{array}\right\}=\left[\begin{array}{cccc}
1 & 0 & 0 & 0 \\
0 & 1 & 0 & 1 / 2 \\
0 & 0 & 1 & 1 / 2 \\
0 & 0 & 0 & 1 / 4
\end{array}\right]\left\{\begin{array}{l}
N_{1} \\
N_{2} \\
N_{3} \\
N_{4}
\end{array}\right\} .
$$

Thus the relevant interpolation is given by

$$
N_{1}=\omega_{1}, \quad N_{2}=\omega_{2}\left(1-2 \omega_{3}\right), \quad N_{3}=\omega_{3}\left(1-2 \omega_{2}\right), \quad N_{4}=4 \omega_{2} \omega_{3}
$$

The reader can then easily verify that this transformed interpolation basis due to the assumed nodal placement is identical to that of the corresponding native simplex element.

Similarly the native transitive element, combining quartic and quintic complete interpolations conformably and having the polynomial completeness of degree four in fig. 11 , is identified with the corresponding adaptive finite element currently used. Thus, in general, we can conclude that the adaptive finite elements have equivalent native simplex members.

It is remarked that the native Lagrange family now produces a variety of higher order simplex elements as well as any transitive elements combining different order interpolations conformably in the conventional shape function form. Furthermore, the variable-node expres- 


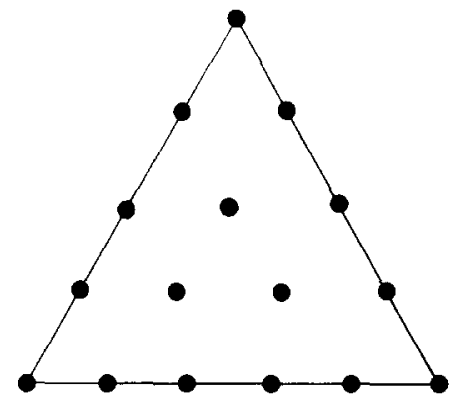

Fig. 11. Typical transitive finite element, which combines the quartic and quintic complete triangles conformably with the polynomial completeness of degree four.

sions as shown in eq. (41) enable us to utilize such harmonious combinations quite easily and systematically.

\section{Generalization of the mapping function space}

We have thus developed our general simplex interpolations in the volume coordinates. Namely, our trial function space for an $n$-node element can be written

$$
\mathscr{F}=\left\{f: E_{\mathscr{F}}(f)=f-\sum_{i=1}^{n} f_{i} N_{i}=0\right\},
$$

for the interpolation of the form

$$
\phi=\sum_{i=1}^{n} \phi_{i} N_{i}\left(\omega_{1}, \omega_{2}, \omega_{3}\right) \text {. }
$$

In the preceding sections, for simplicity, we have tacitly utilized the parametric representation

$$
x=\sum_{i=1}^{3} x_{i} \omega_{i}
$$

where

$$
\boldsymbol{x}^{\prime}=[x, y], \quad \boldsymbol{x}_{i}^{\prime}=\left[x_{i}, y_{i}\right]
$$

Here $x_{i}$ denotes the coordinate vector due to vertex $i$ of 3-node triangle in the global Cartesian system $(x, y)$. Thus eq. (57) redefines in terms of $(x, y)$ the trial function space $\mathscr{F}$ originally characterized by $\left(\omega_{1}, \omega_{2}, \omega_{3}\right)$ in eq. (55).

In order to apply our native simplex interpolations more widely, let us generalize the parametric representation to

$$
x=\sum_{i=1}^{m} x_{i} M_{i}\left(\omega_{1}, \omega_{2}, \omega_{3}\right) .
$$


The mapping shape functions $M_{i}$, by definition, satisfy

$$
M_{i}(j)=\delta_{i j}
$$

Hence the mapping interpolation basis $\boldsymbol{M}=\left\{\boldsymbol{M}_{i}\right\}$ uniquely defines a mapping function space

$$
\mathcal{M}=\left\{f: E_{\mathcal{M}}(f)=f-\sum_{i=1}^{m} f_{i} M_{i}=0\right\} .
$$

In this generalization, the volume coordinate system $\left(\omega_{1}, \omega_{2}, \omega_{3}\right)$ should be regarded as only the local parametric system, satisfying

$$
0 \leq \omega_{i} \leq 1, \quad \sum_{i=1}^{3} \omega_{i}=1 .
$$

However, in this paper, we continue to call the set of $\omega_{i}$ the volume coordinates.

Evidently our native simplex interpolations are applicable to the mapping interpolation basis $M$ also. If we keep the identity of the mapping function space $\mathcal{M}$ and the trial function space $\mathscr{F}$, then any curved iso-parametric elements are derivable just as with the rectangular finite elements. Selecting the appropriate mapping function space, we can thus apply our native simplex family to a variety of problems, an example of which is given in a later section.

\section{Comparison with the rectangular polynomial interpolations with collapse into triangle}

We consider here the cube of fig. $12 \mathrm{~b}$ normalized to $(-1,1)$ in another local parametric system $(\xi, \eta)$ with collapse of nodes 1 and $1_{D}$, to give the triangle of fig. 12a in the global
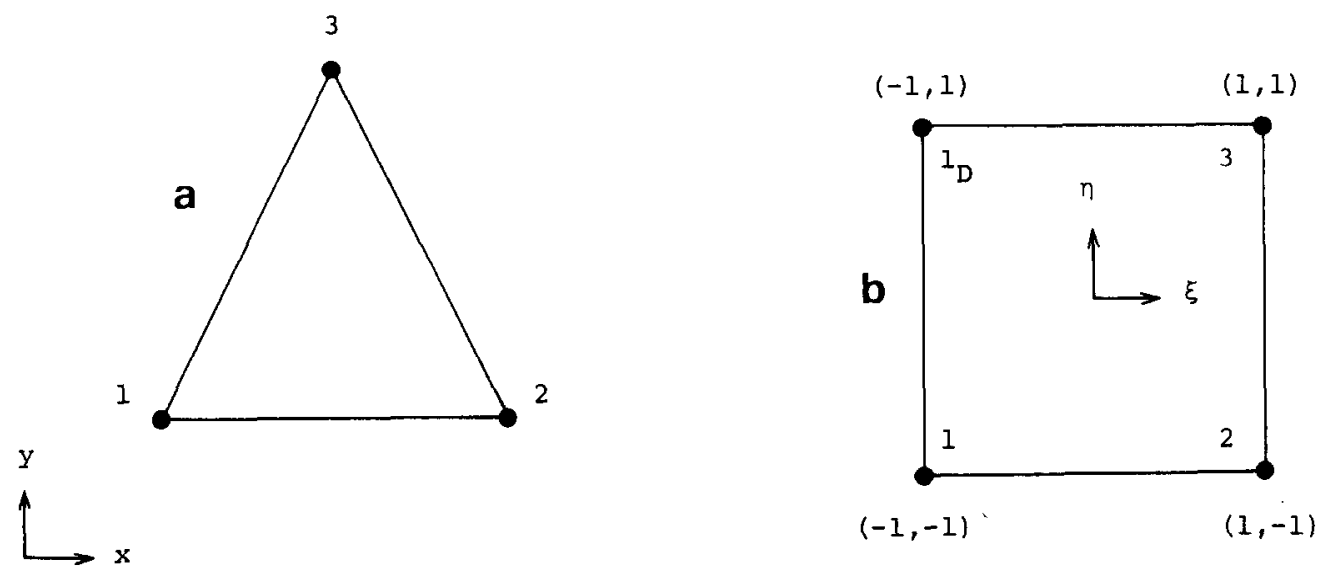

Fig. 12. Simple triangular element geometry; (a) triangle in the global Cartesian system $(x, y)$, (b) normalized cube in the local parametric system $(\xi, \eta)$ with collapse of nodes 1 and $1_{D}$. 
Cartesian system $(x, y)$. Then the collapsed bilinear trial function can be expressed

$$
\phi=\sum_{i=1}^{3} \phi_{i} N_{i}(\xi, \eta)
$$

where

$$
\begin{aligned}
& N_{1}=(1-\xi)(1-\eta) / 4+(1-\xi)(1+\eta) / 4=(1-\xi) / 2, \\
& N_{2}=(1+\xi)(1-\eta) / 4, \quad N_{3}=(1+\xi)(1+\eta) / 4
\end{aligned}
$$

We further prescribe the mapping function space to be iso-parametrically identical to the trial function space; this can be written

$$
\boldsymbol{x}=\sum_{i=1}^{3} x_{i} M_{i}(\xi, \eta)
$$

where

$$
M_{i}=N_{i}, \quad(i=1,2,3) .
$$

Then evidently our trial function space can be uniquely characterized by a monomial basis $[1, x, y]$, and hence this element is the classical, constant derivative triangle $[4,16]$.

We can thus treat our native simplex interpolations also in the local parametric system $(\xi, \eta)$ through the relation

$$
\xi=1-2 \omega_{1}, \quad \eta=\left(\omega_{3}-\omega_{2}\right) /\left(\omega_{3}+\omega_{2}\right) .
$$

For example, the trial function of the famous quadratic triangle with linearly varying derivatives [6] of fig. 13 can be written

$$
\phi=\sum_{i=1}^{6} \phi_{i} N_{i}(\xi, \eta)
$$

where

$$
\begin{aligned}
& N_{1}=\omega_{1}\left(2 \omega_{1}-1\right), \quad N_{2}=\omega_{2}\left(2 \omega_{2}-1\right), \quad N_{3}=\omega_{3}\left(2 \omega_{3}-1\right), \\
& N_{4}=4 \omega_{1} \omega_{2}, \quad N_{5}=4 \omega_{2} \omega_{3}, \quad N_{6}=4 \omega_{3} \omega_{1} .
\end{aligned}
$$

Substitution of eq. (67) into eq. (69) gives

$$
\begin{aligned}
& N_{1}=-\xi(1-\xi) / 2, \quad N_{2}=-(1+\xi)(1-\eta)(1-\xi+\eta+\xi \eta) / 8 \\
& N_{3}=-(1+\xi)(1+\eta)(1-\xi-\eta-\xi \eta) / 8, \quad N_{4}=\left(1-\xi^{2}\right)(1-\eta) / 2, \\
& N_{5}=(1+\xi)^{2}\left(1-\eta^{2}\right) / 4, \quad N_{6}=\left(1-\xi^{2}\right)(1+\eta) / 2 .
\end{aligned}
$$



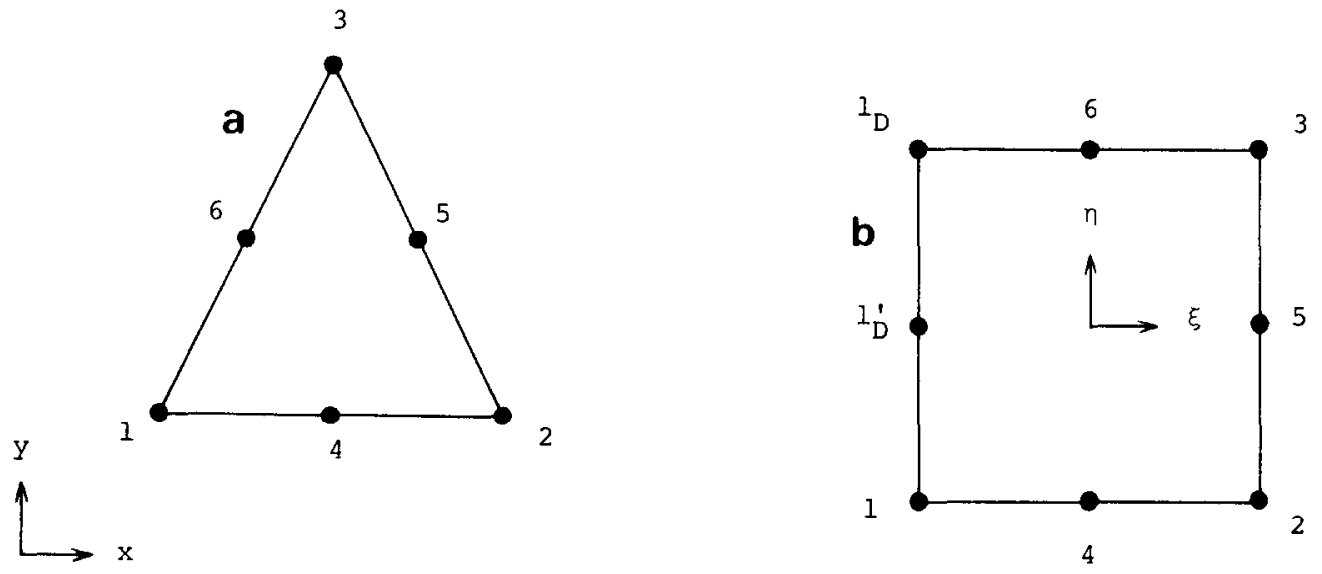

Fig. 13. Quadratic 6-node element geometry; (a) straight-sided triangle in the global Cartesian system $(x, y)$. In our semi-radial singularity mapping, side nodes 4 and 6 are not placed on the centers of corresponding edges. (b) Normalized square with collapse of nodes $1,1_{D}$ and $1_{D}^{\prime}$ in the local parametric system $(\xi, \eta)$.

We note that our native simplex interpolations are polynomials in $(\xi, \eta)$ as well as in $\left(\omega_{1}, \omega_{2}, \omega_{3}\right)$. Furthermore, under the parametric representation by eqs. (65) and (66), this element fulfils polynomial completeness of degree two in $(x, y)$ and $\left(\omega_{1}, \omega_{2}, \omega_{3}\right)$ but not in $(\xi, \eta)$.

On the other hand, the famous serendipity interpolation with collapse can be expressed

$$
\begin{aligned}
& N_{1}=-\xi(1-\xi) / 2, \quad N_{2}=-(1+\xi)(1-\eta)(1-\xi+\eta) / 4, \\
& N_{3}=-(1+\xi)(1+\eta)(1-\xi-\eta) / 4, \quad N_{4}=\left(1-\xi^{2}\right)(1-\eta) / 2, \\
& N_{5}=(1+\xi)\left(1-\eta^{2}\right) / 2, \quad N_{6}=\left(1-\xi^{2}\right)(1+\eta) / 2 .
\end{aligned}
$$

Substituting eq. (67), eq. (71) can be rewritten

$$
\begin{aligned}
& N_{1}=\omega_{1}\left(2 \omega_{1}-1\right), \quad N_{2}=\omega_{2}\left\{2 \omega_{2}-1-2 \omega_{3} \omega_{1} /\left(\omega_{2}+\omega_{3}\right)\right\}, \\
& N_{3}=\omega_{3}\left\{2 \omega_{3}-1-2 \omega_{1} \omega_{2} /\left(\omega_{2}+\omega_{3}\right)\right\}, \quad N_{4}=4 \omega_{1} \omega_{2}, \\
& N_{5}=4 \omega_{2} \omega_{3} /\left(\omega_{2}+\omega_{3}\right), \quad N_{6}=4 \omega_{3} \omega_{1} .
\end{aligned}
$$

It is thus clear that this collapsed rectangular interpolation is not the polynomial in $\left(\omega_{1}, \omega_{2}, \omega_{3}\right)$ and lacks the aesthetic appeal of symmetry in the volume coordinates. Moreover, it fails to have polynomial completeness of degree two not just in $(\xi, \eta)$ and $\left(\omega_{1}, \omega_{2}, \omega_{3}\right)$ but also in $(x, y)$. In order to keep the polynomial completeness in $(x, y)$, we must add an internal degree of freedom. Recall that the famous regular Lagrange interpolations for the cube developed by Argyris [1,2] retain the necessary polynomial completeness even after the collapse. The collapsed rectangular interpolations are thus somewhat redundant in comparison to our native simplex interpolations. 


\section{Incorporation of the derivative singularity}

In fracture mechanics, some singularities are needed in the first derivatives of the trial function, say in the stress and strain. Then naturally the mapping function space is to be different from the trial function space, and our native simplex interpolations can be applied only to the field interpolation basis $\boldsymbol{N}$ or only to the mapping interpolation basis $\boldsymbol{M}$. Here we elect to compose our trial function space by the native simplex interpolations. Namely, our objective is to derive the peculiar simplex mapping called the semi-radial singularity mapping, which has been developed only for the rectangular interpolations with and without collapse $[17,18]$.

Consider, for simplicity, the simple 3-node triangle in the global Cartesian system $(x, y)$ with the parametric representation by

$$
x=\sum_{i=1}^{3} x_{i} M_{i}\left(\omega_{1}, \omega_{2}, \omega_{3}\right)
$$

We define the radius $r$ from node 1 by

$$
r=\left\{\left(x-x_{1}\right)^{2}+\left(y-y_{1}\right)^{2}\right\}^{1 / 2} .
$$

Then with the arbitrary singularity constant $\lambda(0<\lambda<1), O\left(r^{\lambda-1}\right)$ singularities are required in the first derivatives of our trial function within the vicinity of node 1 .

Let us consider the simplest trial function of the form

$$
\phi=\sum_{i=1}^{3} \phi_{i} N_{i}\left(\omega_{1}, \omega_{2}, \omega_{3}\right),
$$

where

$$
N_{i}=\omega_{i} \quad(i=1,2,3),
$$

where the constant field reproducibility is expressed as

$$
1=\sum_{i=1}^{3} N_{i}
$$

We introduce here the non-dimensional radius $\rho$ which should take the value zero at node 1 and unity on the edge opposite to the singular node. Expecting $O\left(\rho^{\lambda-1}\right)$ singularities in our derivatives, we impose the $\rho^{\lambda}$-reproducibility by the condition

$$
\rho^{\wedge}=N_{2}+N_{3} \text {. }
$$

Then we have immediately

$$
\rho^{\lambda}=1-\omega_{1} .
$$


In order to assure in our trial function that the $\rho^{\lambda}$ term is of lowest order, except for the constant term, it is further necessary that the $\rho^{-\lambda} N_{2}$ and $\rho^{-\lambda} N_{3}$ terms should be bounded everywhere within the element. Then if $\rho$ is proportional to $r$ at least within the vicinity of node $1, O\left(r^{\lambda-1}\right)$ singularities may sufficiently be attainable.

We further impose the iso-parametrical identity of our mapping and trial function spaces in the limit of $\lambda=1$. Then corresponding to eqs. (77) and (78), the mapping shape functions should satisfy

$$
1=\sum_{i=1}^{3} M_{i}
$$

and

$$
\rho=M_{2}+M_{3}
$$

Substituting eqs. (73) and (80) into eq. (74), the Euclidean radius $r$ can be rewritten

$$
r=\left[\left\{\sum_{i=2}^{3}\left(x_{i}-x_{1}\right) M_{i}\right\}^{2}+\left\{\sum_{i=2}^{3}\left(y_{i}-y_{1}\right) M_{i}\right\}^{2}\right]^{1 / 2} .
$$

Thus, the proportionality of $\rho$ and $r$ within the vicinity of node 1 is assured, provided that the $\rho^{-1} M_{2}$ and $\rho^{-1} M_{3}$ terms are bounded everywhere.

Our mapping space should thus be connected to the trial space by

$$
\left(1-M_{1}\right)^{\lambda}=1-N_{1}, \quad \rho^{-1} M_{2}=\rho^{-\lambda} N_{2}, \quad \rho^{-1} M_{3}=\rho^{-\lambda} N_{3} .
$$

We have explicitly

$$
M_{1}=1-\left(1-\omega_{1}\right)^{1 / \lambda}, \quad M_{2}=\left(1-\omega_{1}\right)^{-1+1 / \lambda} \omega_{2}, \quad M_{3}=\left(1-\omega_{1}\right)^{-1+1 / \lambda} \omega_{3} .
$$

This is the semi-radial singularity mapping in the volume coordinates. The orbits of our non-dimensional radius are illustrated in figs. 14 and 15 .

In the derivation of the Jacobian transformation, however, it is convenient for us to introduce the local parametric system $(\xi, \eta)$ with eq. (67). Then we have

$$
|\boldsymbol{J}|=\{(1+\xi) / 2\}^{-1+2 / \lambda} T / 4 \lambda,
$$

where

$$
\begin{aligned}
& \partial \xi / \partial x=2 \lambda\left(y_{3}-y_{2}\right)\{(1+\xi) / 2\}^{1-1 / \lambda} / T, \\
& \partial \xi / \partial y=-2 \lambda\left(x_{3}-x_{2}\right)\{(1+\xi) / 2\}^{1-1 / \lambda} / T, \\
& \left.\partial \eta / \partial x=-\left\{\left(y_{2}-y_{1}\right)(1-\eta)+\left(y_{3}-y_{1}\right)(1+\eta)\right\}(1+\xi) / 2\right\}^{-1 / \lambda} / T, \\
& \left.\partial \eta / \partial y=\left\{\left(x_{2}-x_{1}\right)(1-\eta)+\left(x_{3}-x_{1}\right)(1+\eta)\right\}(1+\xi) / 2\right\}^{-1 / \lambda} / T .
\end{aligned}
$$




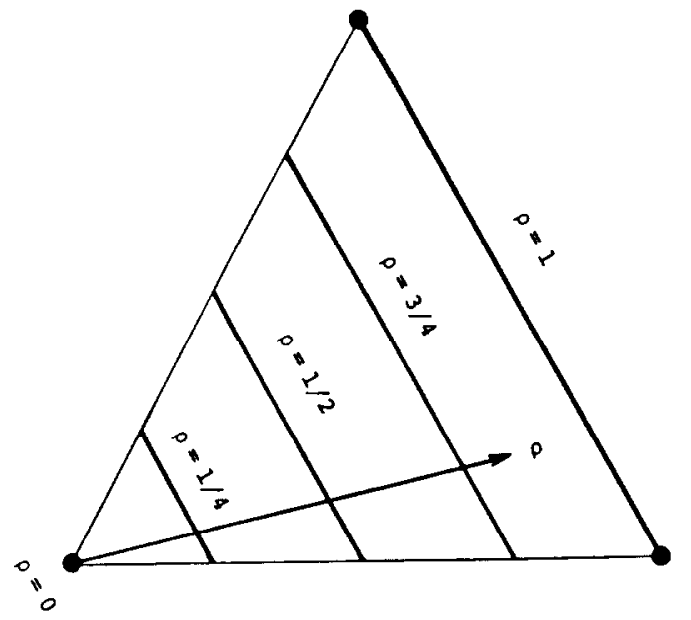

Fig. 14. Constant $\rho$ lines of $\rho^{\lambda}=1-\omega_{1}$ in the global Cartesian system $(x, y)$.

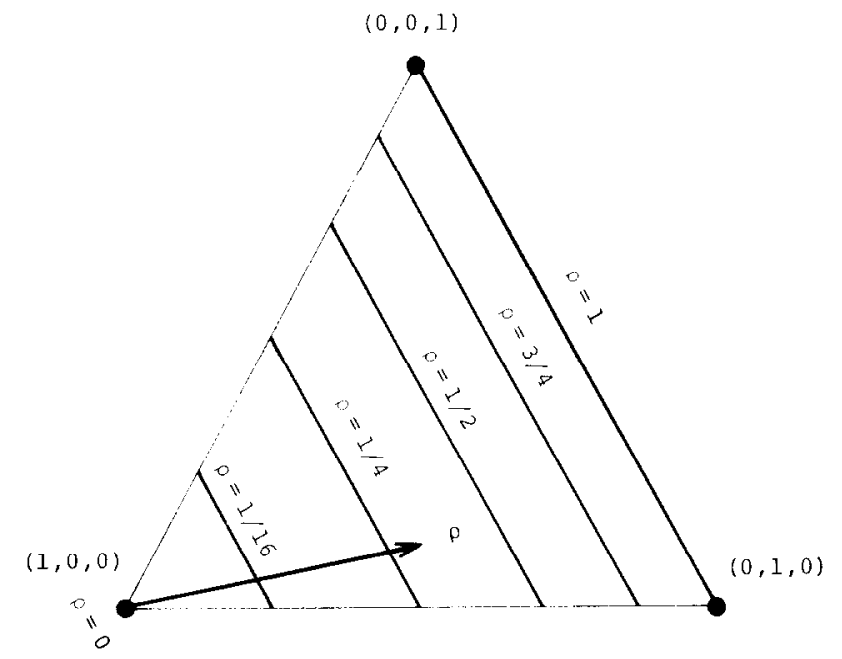

Fig. 15. Typical constant $\rho$ lines with $\lambda=1 / 2$ in the volume coordinates $\left(\omega_{1}, \omega_{2}, \omega_{3}\right)$.

Here $T$ denotes twice the area of the triangle in the global Cartesian system $(x, y)$. For the proof of the realized $\mathrm{O}\left(r^{\lambda-1}\right)$ derivative singularities, see Appendix A.

It is worthy of note that eqs. (67) and (86) enable us to utilize the differentiations of the form

$$
\partial \phi / \partial x=(\partial \xi / \partial x) \sum_{i=1}^{3}\left(\partial \phi / \partial \omega_{i}\right)\left(\partial \omega_{i} / \partial \xi\right)+(\partial \eta / \partial x) \sum_{i=1}^{3}\left(\partial \phi / \partial \omega_{i}\right)\left(\partial \omega_{i} / \partial \eta\right)
$$

Then with eq. (85) the integrations in the practical finite element procedure can be performed in a $(-1,1)$ normalized cube. Since our native simplex interpolations are given in terms of 
volume coordinates, the Gauss-Legendre quadrature can thus advantageously be used just like in the rectangular finite elements.

Once the appropriate mapping shape functions are developed for the original 3-node triangle, we can easily place other additional nodes without changing the original element geometry, and further without affecting the developed transformation formulae. Therefore, all the native simplex Lagrange family members are applicable to the field shape functions with the peculiar singularity mapping in eqs. (73) and (84).

It is further interesting to note that eqs. (85) and (86) are identical to the Jacobian relations developed for the rectangular bilinear interpolation with collapse [18]. Namely the isoparametrical identity between the linear simplex and collapsed bilinear interpolations in the limit of $\lambda=1$ is preserved for arbitrary $\lambda$. However, higher order elements are quite different. For example, the trial function specified by eq. (70) under our semi-radial singularity mapping realizes the reproducibility of the $\rho^{2 \lambda}, \rho^{2 \lambda-2} x^{2}, \rho^{2 \lambda-2} x y$ and $\rho^{2 \lambda-2} y^{2}$ terms, while only the $\rho^{2 \lambda}$ term is reproducible in the trial function by eq. (71). We may have to examine more carefully the observed redundancy of the collapsed rectangular interpolations in fracture mechanics also.

\section{Concluding remarks}

The native Lagrange family for simplex with arbitrarily placed nodes is thus successfully and definitively presented in hierarchy-ranking expressions. This new family produces a variety of higher order elements as well as any transitive finite elements combining different order interpolations conformably. Such harmonious combinations can easily be incorporated into the practical finite element programs with the variable-node expressions. It is further demonstrated that the adaptive finite elements from mixed bases can be identified with some appropriate native simplex members having conventional interpolation bases. Naturally, derivations of the native Lagrange family for $n$-simplex are straightforward.

It is emphasized that the constraints on the placement of side and face nodes are fully relaxed in the native Lagrange family. However, efforts to utilize such generalization without improving the trial function space are meaningless in finite element analysis [11], and hence we must pursue the nodal placement regularity as much as possible in any situation. In applications to other surface generation fields, on the other hand, these relaxations are frequently essential.

In practical finite element applications, it is of great importance to introduce the appropriate mapping function space. As an example in fracture mechanics, we have presented the semi-radial singularity mapping which yields a series of new crack elements with the native simplex interpolations. In particular we emphasize that the trial function spaces of these new singularity elements are quite different from those got from the collapsed rectangular interpolations currently used in fracture mechanics.

\section{Acknowledgment}

The authors are grateful to Prof. N. Takenaka of Nihon University, for his useful comments 
and suggestions. Thanks are also due to I. Nishiguchi and Prof. Y. Yamada of University of Tokyo, for their discussions.

\section{Appendix A. Proof of the occurrence of $O\left(r^{\lambda-1}\right)$ derivative singularities}

We consider generally the trial function over the triangle of the form

$$
\phi=\sum_{i=1}^{n} \phi_{i} N_{i}(\xi, \eta)
$$

Here the field shape functions $N_{i}$ are given by the native simplex interpolations. There, $N_{i}$ is the polynomial in $(\xi, \eta)$ satisfying

$$
1=\sum_{i=1}^{n} N_{i}
$$

Then eq. (A.1) can be rewritten

$$
\phi-\phi_{1}=\sum_{i=2}^{n}\left(\phi_{i}-\phi_{1}\right) N_{i}(\xi, \eta) .
$$

Evidently $N_{i}(i \neq 1)$ should take the value zero at node 1 , and hence $\phi-\phi_{1}$ is such a polynomial as has the $1+\xi$ term, i.e. $\rho^{\lambda}$. The $\partial \phi / \partial \eta$ term thus also has $\rho^{\lambda}$.

Notice here the partial differential formula by

$$
\partial \phi / \partial x=(\partial \phi / \partial \xi)(\partial \xi / \partial x)+(\partial \phi / \partial \eta)(\partial \eta / \partial x) .
$$

Under the required semi-radial singularity mapping in eqs. (73) and (84), eq. (86) shows the $\rho^{1-\lambda} \partial \xi / \partial x$ and $\rho \partial \eta / \partial x$ terms to be polynomials in $(\xi, \eta)$. Thus the singularities in $\partial \phi / \partial x$ are manifestly $O\left(\rho^{\lambda-1}\right)$, i.e. $O\left(r^{\lambda-1}\right)$ within the vicinity of the singular node as expected.

We next prove the boundedness of the strain energy within the element. Obviously the strain energy as well as stiffness matrix components can be estimated by

$$
E=\int_{-1}^{1} \int_{-1}^{1}(\partial \phi / \partial x)^{2}|J| \mathrm{d} \xi \mathrm{d} \eta
$$

Eq. (85) describes that the Jacobian $|J|$ is composed of only the $\rho^{2-\lambda}$ term. Therefore, the integrand of eq. (A.5) is the polynomial in $(\xi, \eta)$ having the $\rho^{\lambda}$ term. Thus we have no singularity in our integrand, and consequently the strain energy is bounded.

It is emphasized that we have $\mathrm{O}\left(r^{\lambda-1}\right)$ derivative singularities with bounded strain energy also for the collapsed rectangular interpolations, so long as the constant field reproducibility (A.2) is satisfied. Evidently the proof presented here is appropriate for any positive $\lambda$. Although the Jacobian vanishes at node 1, the boundedness of stiffness matrix components is thus guaranteed even at $\lambda=1$. 


\section{References}

[1] J.H. Argyris, K.E. Buck, I. Fried, G. Mareczek and D.W. Scharpf, Some new elements for matrix displacement methods, Proc. 2nd Conference on Matrix Methods in Structural Mechanics, Air Force Inst. Tech., Wright Patterson Air Force Base, OH, 1968.

[2] J.H. Argyris and I. Fried, The LUMINA element for the matrix displacement method, Aero. J. 72 (1968) 514-517.

[3] R.L. Taylor, On completeness of shape functions for finite element analysis, Internat. J. Numer. Meths. Engrg. 4 (1972) 17-22.

[4] O.C. Zienkiewicz, The Finite Element Method (McGraw-Hill, London, 3rd ed., 1977).

[5] M. Okabe, Y. Yamada and I. Nishiguchi, Reconsiderations on rectangular Lagrange families with hierarchyranking bases, Comput. Meths. Appl. Mech. Engrg. 23 (1980) 369-390.

[6] J.H. Argyris, Triangular elements with linearly varying strain for the matrix displacement methods, J. Roy. Aero. Soc. 69 (1965) 711-713.

[7] J.H. Argyris, I. Fried and D.W. Scharpf, The TET20 and the TEA8 elements for the matrix displacement method, Aero. J. 72 (1968) 618-625.

[8] P. Silvester, Higher order polynomial triangular finite elements for potential problems, Internat. J. Engrg. Sci. 7 (1968) 849-861.

[9] P.G. Ciarlet and P.A. Raviart, General Lagrange and Hermite interpolation in $\mathbb{R}^{n}$ with applications to finite element methods, Arch. Rat. Mech. Anal. 46 (1972) 177-199.

[10] A.R. Mitchell and R. Wait, The Finite Element Method in Partial Differential Equations (Wiley, London, 1977).

[11] M. Okabe, Y. Yamada and I. Nishiguchi, Basis transformation of trial function space in Lagrange interpolation, Comput. Meths. Appl. Mech. Engrg. 23 (1980) 85-99.

[12] A. Pasini, A. Peano, R. Riccioni and L. Sardella, A self-adaptive finite element analysis, IKOSS Cong., Baden, 1977.

[13] I.N. Katz, A.G. Peano and M.P. Rossow, Nodal variables for complete conforming finite elements of arbitrary polynomial order, Comput. Meths. Appls. 4 (1978) 85-112.

[14] A. Peano, A. Pasini, R. Riccioni and L. Sardella, Adaptive approximations in finite elemcnt structural analysis, Comput. and Structures 10 (1979) 85-112.

[15] K.J. Bathe and E.L. Wilson, Numerical Methods in Finite Element Analysis (Prentice-Hall, Englewood Cliffs, NJ, 1976).

[16] B.M. Irons, A technique for degenerating brick-type isoparametric elements using hierarchical midside nodes, Internat. J. Numer. Meths. Engrg. 8 (1973) 203-209.

[17] Y. Yamada, Y. Ezawa, I. Nishiguchi and M. Okabe, Reconsiderations on singularity or crack tip elements, Internat. J. Numer. Meths. Engrg. 14 (1979) 1525-1544.

[18] M. Okabe, Fundamental theory of the semi-radial singularity mapping with applications to fracture mechanics, Comput. Meths. Appl. Mech. Engrg. 26 (1981) 53-73. 